\title{
Effects of Solar Array Shadowing on the Power Capability of the Interim Control Module
}

James Fincannon and Jeffrey S. Hojnicki

Glenn Research Center, Cleveland, Ohio

James Christopher Garner

Naval Research Laboratory, Washington, D.C.

Prepared for the

34th Intersociety Energy Conversion Engineering Conference sponsored by the Society of Automotive Engineers

Vancouver, British Columbia, Canada, August 1-5, 1999

National Aeronautics and

Space Administration

Glenn Research Center 


\section{Acknowledgments}

Of course, performing quality engineering analysis for such complex systems often requires quality data from a number of outside sources. The authors would like to thank John Anderson of Boeing for compiling the comprehensive listing of solar array and radiator gimbal modes and lock angles for all ISS stages; Bob Hensheid and his team at Boeing for supplying the ISS Torque Equilibrium Attitudes, Brian Richard of the Johnson Space Center

MAGIK Team for their ICM placement options, and the Lockheed Martin's SEMDA Lab for their detailed geometric models and graphical depictions of ISS for all assembly flights. The authors also wish to acknowledge the efforts of the many talented people who have contributed to the development of SPACE over the years, and the good folk at the ISS Program Office, Boeing, Boeing-Canoga

Park and Lockheed for their various disparate efforts that have made the many pieces a whole.

Trade names or manufacturers' names are used in this report for identification only. This usage does not constitute an official endorsement, either expressed or implied, by the National Aeronautics and Space Administration.

Available from

NASA Center for Aerospace Information 7121 Standard Drive

Hanover, MD 21076

Price Code: A03
National Technical Information Service 5285 Port Royal Road Springfield, VA 22100 Price Code: A03 


\title{
Effects of Solar Array Shadowing on the Power Capability of the Interim Control Module
}

\author{
James Fincannon, Jeffrey S. Hojnicki \\ National Aeronautics and Space Administration \\ Glenn Research Center
}

James Christopher Garner

Naval Research Laboratory

Washington, D.C.

\begin{abstract}
The Interim Control Module (ICM) is being built by the US Naval Research Laboratory (NRL) for NASA as a propulsion module for the International Space Station (ISS). Originally developed as a spinning spacecraft used to move payloads to their final orbit, for ISS, the ICM will be in a fixed orientation and location for long periods resulting in substantial solar panel shadowing. This paper describes the methods used to determine the incident energy on the ICM solar panels and the power capability of the electric power system (EPS). Applying this methodology has resulted in analyses and assessments used to identify ICM early design changes/options, placement and orientations that enable successful operation of the EPS under a wide variety of anticipated conditions.
\end{abstract}

\section{INTRODUCTION}

The ICM is being built by the US Naval Research Laboratory for NASA as a propulsion module for the ISS [1]. Its purpose on ISS is to augment or replace the Service Module, providing reboost and attitude control functions. As originally developed, the ICM was used to move payloads to their final orbit and provide attitude control, while operating as a spinning spacecraft. On the ISS, the ICM will operate differently. It will be attached to the station in a fixed orientation for long periods. Surrounded by large ISS structures, such as solar arrays and radiators, the much smaller ICM will be subjected to severe thermal impacts and solar panel shadowing.

To ensure the ICM EPS will produce sufficient power, it was necessary to assess the ICM performance in a variety of ISS configurations, flight modes and orbit conditions. Engineers at NASA Glenn Research Center (GRC) and NRL collaborated to perform those assessments. Detailed solid models of the ICM exterior geometry (and its 16 ICM solar panels), Space Shuttle Orbiter and ISS were needed. Flight dynamics analyses were also needed to determine the ISS attitude for each configuration and flight mode. NASA GRC engineers then input those data into their SPACE computer model, which predicted time-varying shadow patterns and the total incident solar energy on each ICM solar panel. Incident energy values were used to identify flight modes and configurations that required further analysis, but an ICM EPS model was needed to determine whether energy balance was achieved. NRL engineers input the SPACE incident energy results into their energy balance tools to determine the power capability of the ICM. Electrical loads analyses then allowed the power margin to be calculated. The culmination of all these linked analyses was an assessment of the feasibility of utilizing the ICM on the ISS, as well as an optimized design and location.

This paper describes methods used to determine the incident energy on the ICM solar panels and the EPS power capability. Results from selected assessments are presented, which were used to finalize ICM design changes, and to determine the optimum placement and orientation of the ICM on the ISS.

There were three phases to this assessment. Phase 1 was an initial study of the ICM, aft of the FGB (or Zarya Module) as a candidate replacement the Russian Service Module, in case it could not be completed. Phase 2 considered a later addition of the ICM, aft of the Service module. In this case, it would augment the Service module capabilities and enhance overall reliability. Phase 3 examined another option in which it would be placed on the US side of ISS. Here it would also augment the Service Module, but this placement would reduce interfaces between US and Russian elements.

\section{METHODS}

The assessment of ICM power capability was composed of two tasks: determination of the incident energy on 
each solar panel, performed by NASA GRC; and assessing the power and energy balance of the ICM, performed by NRL.

\section{DETERMINING INCIDENT ENERGY}

Tool

Incident energy was determined using a computer model called SPACE (System Power Analysis for Capability Evaluation), which was developed for the ISS program by NASA GRC [2-5]. It is a highly-integrated, end-to-end model of the ISS EPS, with 3 distinct operating modes:

1. A "pointing-only" mode, which uses only orbit mechanics and solar array pointing/ shadowing modules. This mode has been used extensively to provide data to ISS participants such as Khrunichev Space Center and the European Space Agency.

2. A "source-driven" mode, which predicts the maximum power level that the EPS can sustain through an orbit, for a set of conditions.

3. A "load-driven" mode, which determines the ability of the EPS to supply a specified time-varying load demand, under a set of operational constraints.

Only the first mode was used for the analyses presented in this paper.

To calculate incident energy, SPACE: 1) determines the orbit conditions, 2) determines the gimbal angles for each ISS solar array and radiator, 3) assembles the ISS geometry, 4) determines the shapes and sizes of any shadowed area on surfaces of interest (i.e. ICM solar panels), and 5) calculates the amount of incident solar energy each panel receives, based on any shadowed area and the orientation of the panel with respect to the sun. Some of the important SPACE modules are described below.

\section{Orbit Mechanics}

SPACE contains an integrated orbit mechanics module which can model circular earth orbits. Critical parameters determined by this module include the insolation and eclipse times and the solar beta ( $\beta$ ) angle for the orbit. The solar $\beta$ angle is the angle between the Earth-sun vector and the plane of the ISS orbit. This angle is crucial to determining the orientation of the ICM solar panels with respect to the sun. Data are generated in approximately one minute time steps, through the nominally 90 minute orbit.

\section{Gimbal Algorithms}

SPACE models each ISS gimbal based on the actual hardware constraints and control algorithms employed. For instance, solar array gimbals on the Zarya module do not continuously rotate through $360^{\circ}$. Rather, they move in a ratcheting motion and contain hard-stops, thus requiring unwinding of the gimbals at certain times. US solar arrays, in contrast, can continuously rotate through $360^{\circ}$, and are commanded based on an on-orbit calculation of the sun vector. SPACE models each of these features as they apply to each gimbal on the ISS.

\section{Geometry \& Shadowing Algorithms}

The shadowing and incident energy module is critical to this analysis. [6-7] In SPACE, the shadowing algorithms account for the changing ISS geometry during assembly. This module generates the geometric information associated with a particular ISS configuration (including rotating components like solar arrays, and visiting vehicles such as the Soyuz or Space Shuttle Orbiter). The module also determines the orientation of the ISS with respect to the sun. With this information, the shapes and areas of any shadows on the solar panels is calculated. Because of its integrated capability to determine detailed shadow patterns, the SPACE shadowing module has been applied in a variety of applications, including proposed solar dynamic systems on Mir and ISS; solar arrays on various ISS elements, such as the Zarya Module, Service Module, Science Power Platform and Automated Transfer Vehicle; and other proposed ISS payloads.

\section{SPACE Validation}

The "pointing-ony" mode of SPACE was validated during ISS Verification Analysis Cycle \#1, which was completed in January, 1998. The remaining modes will be validated in preparation for future ISS analysis cycles.

Inputs

\section{Geometry}

The geometric configuration of the ISS, including visiting spacecraft, is an essential input. Each ISS object must be associated with a launch in the assembly sequence as well as a physical location. The Revision C Baseline assembly sequence was used throughout this analysis. Lockheed Martin's Systems Engineering and Modeling and Design Analysis Laboratory (SEMDA) maintains highly detailed IDEAS ${ }^{2}$ 3D solid models of each of the stages in the assembly sequence. These data are simplified by NASA GRC using $3 D$ Studioß to minimize the number of polygons, resulting in ISS models on the order of 1000 polygons. Figure 1 shows the entire ISS geometry with labeled primary elements.

Figure 2 illustrates the simplified ICM geometry that was obtained from NRL. The geometry includes primarily the 16 solar panels ( 12 on the sides and 4 on the aft end), 2 thruster arms, 2 fuel tanks, docking ports and body 
structure. The spacecraft is approximately 16 feet long and 12 feet in diameter. An extended side solar panel option has the arrays increased in length by $40 \%$.

Other necessary geometry includes the location and orientation of the ICM with respect to the other ISS elements. Phase 1 placed the ICM on the Russian side of the ISS, aft of the Zarya Module, while phase 2 examined the ICM aft of the Service Module. Phase 3 examined a number of possible locations on the US side of the ISS, including locations while the ICM was actively controlling the station, as well as stowage locations. These locations included both port ( $Y$ direction) and nadir ( $+Z$ direction) of Node 1, forward of the US Lab Module and forward of Node 2. The separate stowage location is needed since the ICM would use an Orbiter docking port during reboost operations. Figure 3 shows the ICM orientations for each of the Phases.

\section{Gimbals}

A number of structures on the ISS are gimbaled, which means that they rotate with respect to the station core. Thermal radiators and solar arrays, on both the US and the Russian sides of ISS, are the predominant rotating structures. Typically, the solar arrays are commanded to track the sun, using either single or dual-axis tracking. Radiators typically are rotated to maintain an orientation edge-on to the sun. However, there are many activities that prevent nominal gimbal tracking for structural, contamination, or clearance reasons. These can include maintenance operations, reboost, orbiter docking, or even orbits at very high solar $\beta$ angles. The tracking of each of these structures is vital to calculating the shadowing on the ICM solar panels. SPACE is programmed to handle any array or radiator tracking method that the on-orbit control system can perform. This information is input to SPACE before each run, depending on the configuration and operating mode being studied.

These inputs are derived from a comprehensive listing of solar array and radiator tracking modes and lock angles that has been compiled by Boeing.

\section{Attitude}

Another critical input for the analysis is the attitude of the ISS during the orbit, since this directly impacts the orientation of the ICM with respect to the sun, and the shadowing that the ICM will see from other structures. There are several flight attitudes baselined for ISS operation, depending on the ISS configuration and orbital condition. The ISS coordinate system is shown in Figure 1 , with the $+X$-axis in the velocity vector and the $+Z$-axis pointing in the nadir direction (towards earth). This is also the nominal attitude of the ISS and is commonly referred to as the XvvZnadir flight mode. For a variety of reasons, early in the life of the ISS, it is advantageous to operate in some other attitudes. These include ax XnadirSpin mode in which the station $+X$-axis is oriented toward the nadir, and the entire station is spun slowly about that $X$-axis. Another important flight attitudes is known at Xpop. In this attitude, the ISS +X-axis is oriented perpendicular to the orbit plane, while the other two axes are help inertial with respect to the sun. Xpop provides increased power production from US and Russian solar arrays at high solar $\beta$ angles in some of the early station configurations. Another configuration to note is the attitude during reboost operations. Normally, reboost occurs in the XvvZnadir flight mode, but in some cases (Phase 3) examined in this study, reboost occurred with the $-X$-axis in the velocity vector.

For each attitude and configuration, the ISS often flies in a torque equilibrium attitude (TEA). This is the an offset to the pure attitude that minimizes propellant usage. Although these offsets are normally small (less than $10^{\circ}$ for most configurations, with the exception of the pitch offset with the Orbiter docked), they can still effect the shadowing on the ICM and thus must be accounted for in the analysis. A team at Boeing determines the TEAs for each configuration and flight mode and provides that data to GRC.

\section{Limitations}

Although SPACE is one of the most highly-integrated and comprehensive power system models ever built, it nevertheless contains some simplifying assumptions that must be understood to correctly interpret these analysis results. First and foremost, traditionally, the affects of Earth albedo (solar energy reflected off the Earth's surface) on solar array power production has been ignored. This was due to the fact that the US solar arrays are sun-pointing the majority of the time, with only the back sides receiving albedo illumination. Power production from the back side of the US arrays is significantly less efficient than that from the front side, and the magnitude of the albedo flux is highly variable (dependant on factors such as weather and latitude) and difficult to accurately predict. Thus, neglecting the albedo power production has been a prudent, conservative assumption. However, the ICM's orientation will result in some of its solar panels to be pointed directly at the earth in some attitudes, resulting in a noticeable increase in ICM power production. However, the total ICM energy predicted will likely be underestimated by no more than $10 \%$ with most at lower absolute solar $\beta$ angles. Similarly, reflections off of other ISS surfaces are ignored, with an estimated impact of $<2 \%$ on the predicted incident energy.

In addition, SPACE assumes the Sun is a point source, and ignores the size of the disk (half angle $=.266^{\circ}$ ). In reality, shadows are not step changes in intensity, but 
rather gradual intensity variations. The effect is minimal for shadowing objects near a solar panel, but can be more noticeable for shadowing objects far from the panel. The underestimation is as much as $5 \%$ for cases with transient shadowing.

\section{ASSESSING ICM ENERGY BALANCE}

\section{$\underline{\text { Tool }}$}

The NRL power generation model calculates the power capability of the ICM arrays and the resulting power balance when power consumption is included. The general method is to calculate how much energy is required to recharge the battery based on the amount of load drawn during eclipse. If the amount of energy provided by the solar array is insufficient to recharge the battery and provide energy for insolation loads, then a negative margin results. A negative margin indicates that the batteries cannot be recharged in the orbit and will, over a certain number of orbits, eventually discharge depending on the magnitude and duration of the negative margin.

\section{Inputs}

The following data is utilized by the ICM energy balance sottware tool to determine the energy provided by the solar arrays.

1. Solar panel efficiency (using solar array manufacturer data).

2. Average flux.

3. Area of solar cells (for each panel).

4. Incident energy flux data from NASA GRC (for each panel) accounting for shadowing and cosine loss.

The ICM loads database is used to determine which loads are active for the flight in stowed and reboost configurations and total the energy utilization for the orbit. There are approximately 35 loads in the ICM. Loads for the ICM go from $467 \mathrm{~W}$ average to $575 \mathrm{~W}$ peak. The primary difference is due to the activation of heaters.

\section{Limitations}

Because of the complexities involved in linking models, the ICM solar array wiring (cell connectivity) is not modeled. Therefore, the power loss is equal to area shadowed. In the normal implementation of SPACE for the US solar arrays, the shadowing of each cell submodule is accounted for in the string of cell submodules. Shadowing of a significant fraction of the cell submodules on a string can result in the entire string being shut down because of its inability to provide the necessary voltage. For US solar arrays, as little as $25 \%$ of the cell submodules being shadowed can shut down a string. It was felt that in the ICM case, this effect is not as significant because most shadowing on panels was large and rapid. An early analysis (stages $2 A-8 A$, range of solar $\beta$ angles, TEAs and flight modes) of string directionality effects on the ICM panels proved that the strings were oriented optimally for the ICM orientation at the reboost and nadir locations. String effects could especially show up with long narrow shadowing surfaces like the thruster arm. Orbit average incident energy overestimation of less than $10 \%$.

Another limitation of the analysis is that because of the transient high amounts of shadowing, there may be times when the shadowing causes the solar array power to drop below the required load power, thus causing the battery to discharge. Not accounting for this in the energy balance analysis may cause the power to be overestimated by $10 \%$.

\section{RESULTS}

Literally hundreds of individual cases had to be examined to assess the performance of the ICM in each proposed ISS location. For each location, several flight attitudes were examined, both with and without the Space Shuttle Orbiter mated to the station. Special operating modes, such as Orbiter docking and ISS reboost also needed to be studied, and each case was examined over a range of solar $\beta$ angles from $+70^{\circ}$ to $-70^{\circ}$. Finally, that complete set of cases was duplicated for a number of ISS configurations throughout the assembly sequence. Some options to increase energy production were also considered, particularly, adding or enlarging ICM solar array panels.

This paper presents a sampling of the numerous results produced. A sample assessment from each phase of the analysis is presented, consisting of several different flight modes. Both incident energy and the energy balance results are presented in summary plots as a function of solar $\beta$ angle.

\section{INCIDENT ENERGY RESULTS}

SPACE generates detailed plots of the ISS geometry and orientation, as well as plots showing the shadow patterns on each ICM solar panel, throughout each orbit analyzed. Figure 4 shows a sample image of the ISS orientation and shadow patterns as produced by SPACE for a single time step. The left half of the figure shows an image of the ISS, as seen from the sun. The rectangles in the middle of the figure represent each solar panel black areas are shadows, gray areas are sunlit. The rectangles correspond to panels as shown on the right ICM diagram with the upper 3 panels on each thruster side ( 2 sides have these arms), 3 panels on each fuel tank side ( 2 sides have tanks), 4 aft rectangle panels and 4 aft corner segments. 
To provide data to NRL that can be used in their models, these detailed data must be summarized. This is accomplished by calculating the incident energy fraction on each solar panel:

$$
\mathrm{IE}=\cos (\theta)^{*}(1-\mathrm{S})
$$

Where $\theta$ is the angle between the solar panel normal and the sun vector, $S$ is the fraction of the panel area that is shadowed, and IE is the instantaneous incident energy fraction.

This instantaneous incident energy fraction is integrated with respect to time, to give a total incident energy fraction, and provided to NRL for their analysis.

To perform quick assessments as to the viability of each case, another figure of merit is calculated, which is referred to as total relative incident energy (TRIE). This is calculated by summing the product of the incident energy fractions multiplied by each panel's relative surface area. Although this is not a direct measure of EPS capability (since it does not include the solar cell efficiency, solar cell packing factors or active cell area.), it provides a first-look quantitative comparison between cases, prior to the NRL energy balance calculations. Based on NRL results, typically, any average incident energy fractions below 2.0 for the stowed configuration or 3.0 for reboost (because of the different load requirements) may have energy balance shortfalls.

To illustrate the necessity of accounting for ISS structures in assessing the incident energy and energy balance for the ICM, a comparison case was performed in which ICM energy analyses were performed with and without the ISS structure. The case shown is for ISS stage 10A (Figure 4 shows this configuration) which has one port and one starboard U.S. photovoltaic power module, and part of the Russian Science Power Platform solar arrays deployed along with various core station hardware. The flight attitude was assumed the same for both cases (XvvZnadir, Yaw $=-2.0^{\circ}, \quad P i t c h=-8.0^{\circ}$ and Roll $=-1.0^{\circ}$ ). The load that the ICM needed to support was based on its stowed, or quiescent state, and not the higher reboost power level. The location of the ICM in this case was at the nadir of Node 1, and it had the standard length solar panels. If analysts had to assess the incident energy on the ICM without a tool like SPACE, they would be able to account for the pointing error of each ICM solar panel and perhaps the shadowing from other ICM hardware. However, the analysts would have great difficulty in determining the shadowing on each ICM solar panel from the ISS structure. This is because the shadows cast by these structures are very complex and continually change with time due to the motion of the ISS solar arrays and orbit motion (varying position of the Sun relative to the ISS). Figures 5 and 6 clearly show that by not considering the shadowing caused by both ISS core structure and solar arrays, an analyst would erroneously determine that the ICM would be energy self sufficient.

Figures 7,9 and 10 show typical results from the Phase 1,2 and 3 incident energy analyses. The data are plotted as functions of solar $\beta$ angle. Figure 7 is a proximity operations flight in which the ICM is attached to the FGB but several types of flight attitudes are considered and STS attached and ISS alone cases are shown. Figure 9 is for the stage 12A ISS configuration with the ICM attached aft of the Service Module (in the $-X$ direction). Figure 10 is for the stage 12A ISS configuration with the ICM attached in two different stowed locations: the nadir side of Node 1, and forward of the U.S. Lab. The latter location is also the reboost site for ICM.

\section{ENERGY BALANCE RESULTS}

Figure 8 shows typical energy balance results for Phase 1 . Figure 11 shows typical energy balance results for Phase 3.

\section{DISCUSSION}

The primary goal for each phase of analysis was to determine, based on all the cases, if power converters were needed to feed power from the $120 \mathrm{~V}$ ISS main bus to the $28 \mathrm{~V}$ ICM system to augment its power. A converter is not favorable option because of the cost and complexity of attaching and detaching such a converter on-orbit (these operations would require an astronaut extravehicular activity). Acceptable options that were considered to eliminate converter usage included larger/more ICM solar panels, ICM power down, ISS attitude change and ICM placement/orientation.

Phase 1 analyses show that for stage 2A.1 proximity operations, the incident energy is very low at low $\beta$ angles in the +Xnadir flight mode. This is mainly due to the Shuttle shadowing the ICM arrays. In the -Xnadir attitude, the incident energy is also low at low solar $\beta$ since the ICM is on the nadir side of ISS. In Xpop mode, with the Shuttle docked to the ICM, the incident energy begins to drop off rapidly at absolute solar $\beta$ angles above $40^{\circ}$ due to shadowing by the Shuttle. An ISS pitch to angles greater than $25^{\circ}$ would extend the viable range beyond $40^{\circ}$ solar $\beta$. Incident energy levels are substantial for all solar $\beta$ angles in Xpop with the Shuttle attached to Node 1 . The results from the stages $3 A-7 A$ analyses showed that in general, incident energy values were high during Xpop flight modes, with the exception of very low $\beta$ angles combined with a positive ISS pitch. Biasing the station to a negative pitch results in a high incident energy value for all solar $\beta$ angles. In $X v v Z$ nadir, there is lower incident energy at low solar $\beta$ angles, especially for flights past $4 \mathrm{~A}$ when shadowing 
from US solar arrays becomes significant. As with Xpop cases, a positive ISS pitch is detrimental to incident energy while negative pitch increases incident energy.

Initial Phase 1 energy balance results showed many cases with negative energy margins. This originally indicated that a power converter was needed between the ISS power system and the ICM. The most obvious solution was to change the pitch of ISS such that the ICM is on the Sun-side (not shadowed by the ISS or Shuttle). Generally, a more negative pitch improved the power capability for Xpop and XvvZnadir. Also, a reevaluation of loads reduced the power demand somewhat, improving the situation. Increasing the solar cell density on aft panels also helped matters. Numerous options of additional solar panels were considered to improve the power situation further. These included panels mounted on the side of the docking port and on the thruster arms, unfoldable solar panels on the aft end and extended side solar panels. The extended solar panels proved to be the most beneficial and feasible option and eliminated most of the negative margin cases. XvvZnadir still posed a problem that could be reduced by changing ISS attitude. Powering down was not an option because, based on the thermal analysis, for every load removed, a heater must be turned on to maintain thermal equilibrium.

Phase 2 analyses showed the following trends. In XvvZnadir, a docked Shuttle reduced the ICM incident energy on average about $12 \%$ for most solar $\beta$ angles. In Xpop, Shuttle attached cases always resulted in lower ICM incident energy (on average about $29 \%$ lower). The results are mainly due to the attitude differences, however, not shadowing caused by the Shuttle. Shadowing was caused by transient passage of ISS structures between the Sun and the ICM solar panels. Xpop provides more incident energy than XVv-Znadir for all cases except solar $\beta$ equal to $0^{\circ}$ without the Shuttle docked, and less than $40^{\circ}$ with the Shuttle (on average about 34\% more energy). Xpop cases have the ICM aft end pointing toward the Sun for non-zero solar $\beta$ angles. There is little or no shadowing due to non-ICM structures even with the large attitude changes caused by the docked Shuttle. Reboost cases were not specifically examined, but the data that are most similar to the reboost configuration show relatively good incident energy metrics. In general, since the apparent incident energy shortfall was small, extended solar array panels could likely be added to provide positive energy balance.

Phase 2 cases were not analyzed from an energy balance perspective because the option was overcome by events.

Phase 3 analyses showed the following trends. In Xpop for stages $9 \mathrm{~A}$ through $12 \mathrm{~A}$, there is a definite incident energy shortfall. Increasing the solar array areas would likely not be beneficial because of the poor Sun access of the ICM solar panels. TEAs for those flights need to modified to accrue any benefit of larger panels. For 7A, incident energy goes to nearly zero. This points to the need for both TEA changes and solar array area increases. In XvvZnadir, the incident energy is relatively ample except at the lower solar $\beta$ angles. Adding more surface area to the solar arrays may increase the incident energy moderately. Unfortunately, extended solar arrays are not an option for this case because they would hinder ISS robotic arm access to the forward end of the ICM. For the Shuttle docked cases especially on flight $11 \mathrm{~A}$, the incident energy is nearly zero. The reason for this is the poor location of the ICM, with much of the ISS between the ICM and the Sun, and the poor orientation of the ICM solar arrays. Flight attitudes cannot be changed in these cases, so power converters/feeds may be needed. For reboost, the ICM has sufficient incident energy if augmented with extended solar panels.

Stowing the ICM at the reboost PMA location and flying in XvvZnadir (for all solar $\beta$ angles) or Xpop (for absolute solar $\beta$ angles $\left\langle 37^{\circ}\right.$ ) results in higher incident energy for the ICM. Stowing the ICM at the reboost PMA location and flying in Xpop for absolute solar $\beta$ angles greater than $37^{\circ}$ results in a definite incident energy shortfall. Increased solar array area cannot address this issue unless the TEAs for those flights are modified. Power converters/feeds may be required. With the Shuttle docked, the ICM must be at the nominal Node 1 stowage location. Incident energy shortfalls are likely for all flights (except 7A.1) in XvvZnadir and low absolute solar $\beta$ angles (less than $10-20^{\circ}$ ). For 7A.1, all negative solar $\beta$ angles may be a problem. For Xpop, incident energy shortages will exist for all flights except 7A.1, which has good incident energy for absolute solar $\beta$ angles above $50^{\circ}$. In all of these STS-docked cases, because of the sizable blockage of the ICM, extended solar arrays may not be able to alleviate the energy shortfall without modified TEAs. Stowing the ICM at the reboost location will extend the time that the ICM can operate significantly without power converters. A power converter is needed for the energy shortfalls when the Shuttle is docked and when the ISS is at Xpop at high absolute solar $\beta$ angles $(>37)$. Stowage of the ICM at the port or nadir locations provides poor incident energy due to the orientation of the solar arrays with respect to the Sun and close proximity to large structures that can induce shadowing.

Phase 3 energy analysis showed the following trends. Because reboost loads are higher than stowed loads, the energy balance trends and analysis differ somewhat from those derived for the incident energy. Analysis of extended solar arrays for Phase 3 cases was dropped because of marginal improvement in wide variety of cases the ICM must encounter. Reboost cases are unacceptable based on negative margins (reboost has high load demands). Placement of the ICM in a worse 
shadowing environment than the aft FGB or aft Service Module cases and using the ICM on later/more densely populated flights have made the energy generation situation much worse and not easy to correct using relatively simple methods such as larger solar arrays. Negative energy balance for a number of flight attitudes indicate that the preferred approach is to utilize a power converter to transfer power from ISS to the ICM for these later flights.

\section{CONCLUSION}

This paper has described the methodology and presented typical results that were obtained in an effort to determine the feasibility and effectiveness of the ICM for use on the ISS. Changing ISS geometry, both in terms of ISS module deployment or relocation and the continuous variation of non-ICM solar array and radiator orientations were included in the analysis. In addition, a range of possible orbital conditions, including yearly variation and ISS flight attitude were considered. All of this information was utilized to assess ICM solar panel shadowing and incident energy capability as well as power balance and energy generation capability. Numerous ICM location and orientation options were considered in order to optimize the ICM operation.

These assessments have been used to identify ICM placement and orientations that enable successful operation of the EPS under various anticipated conditions. The results indicate:

1. For ISS Stages prior to $7 \mathrm{~A}$ (with the ICM aft of the FGB), the energy production is sufficient if the ICM utilizes extended side panel solar arrays.

2. For ISS Stages post 7A (with the ICM aft of the Service Modules), the energy production is sufficient only by using power interface converters or significant TEA changes.

3. For ISS Stages post 7A (with the ICM on the US side), the energy production is maximized by placement of the ICM at the reboost location for stowage, otherwise significant TEA changes and/or power converters are required.

\section{CONTACT}

James Fincannon

NASA Glenn Research Center, 21000 Brookpark Road, MS 500-203, Cleveland, Ohio 44135

Phone: (216) 433-5405, Fax: (216) 433-2995

Email: fincannon@grc.nasa.gov

MSME University of Toledo, BAE Georgia Institute of Technology
Jeffrey S. Hojnicki

NASA Glenn Research Center, 21000 Brookpark Road, MS 500-203, Cleveland, Ohio 44135

Phone: (216) 433-5393, Fax: (216) 433-2995

Email: jeffrey.s.hojnicki@grc.nasa.gov

MSME and BSME, University of Toledo

James Christopher Garner

Naval Research Laboratory, Code 8134, 4555 Overlook

Ave. SW, Washington, D.C. 20375

Phone: (202) 767-9075, Fax: (202) 767-4633

Email: garner@ssdd.nrl.navy.mil

\section{REFERENCES}

1. The International Space Station Interim Control Module EPS, W. E. Baker, US Naval Research Laboratory, IECEC-98-247, 33rd Intersociety Engineering Conference on Energy Conversion, Colorado Springs, $\mathrm{CO}$, August 26,1998

2. Space Station Freedom Electrical Performance Model, Hojnicki, J. S., Green, R. D., Kerslake, T. W., McKissock, D. B., Trudell, J. J., NASA TM-106395, 28th Intersociety Energy Conversion Engineering Conference, Allanta, Georgia, August 8-13, 1993.

3. System Performance Predictions For Space Station Freedom's Electrical Power System, Kerslake, T. W., Hojnicki, J. S., Green, R. D., Follo, J. C., NASA TM106396, 28th Intersociety Energy Conversion Engineering Conference, Atlanta, Georgia, August 8-13, 1993.

4. Analysis of Shadowing Effects on Spacecraft Power Systems, Fincannon, J., NASA TM-106994, Fourth European Space Power Conference, Poitiers, France, September 4-8, 1995.

5. Load-Following Power Timeline Analyses for the International Space Station, Fincannon, J., Delleur, A., Green, R. D., Hojnicki, J. S., NASA TM-107263, 31th Intersociety Energy Conversion Engineering Conference, Washington D.C., August 11-16, 1996.

6. Analysis of Solar Receiver Flux Distributions for US/Russian Solar Dynamic System Demonstration on the MIR Space Station, Kerslake, T. W. and Fincannon, J., NASA TM-106933, 30th Intersociety Energy Conversion Engineering Conference, Orlando, Florida, July 31-August 4, 1995.

7. Analysis of Shadowing Effects on MIR Photovoltaic and Solar Dynamic Power Systems, Fincannon, J., NASA TM-106940, 30th Intersociety Energy Conversion Engineering Conference, Orlando, Florida, July 31-August $4,1995$.

\section{ACRONYMS}

\section{EPS:}

Electrical Power System

FGB:

Functional Control Block or Zarya 
GRC:

NASA Glenn Research Center

ICM:

Interim Control Module

ISS:

International Space Station

\section{MAGIK}

Manipulator Analysis, Graphics, and Integrated
NRL:

Naval Research Laboratory

SPACE:

System Power Analysis for Capability Evaluation

\section{SEMDA:}

Systems Engineering and Modeling and Design Analysis Laboratory

TEA:

Torque Equilibrium Attitude

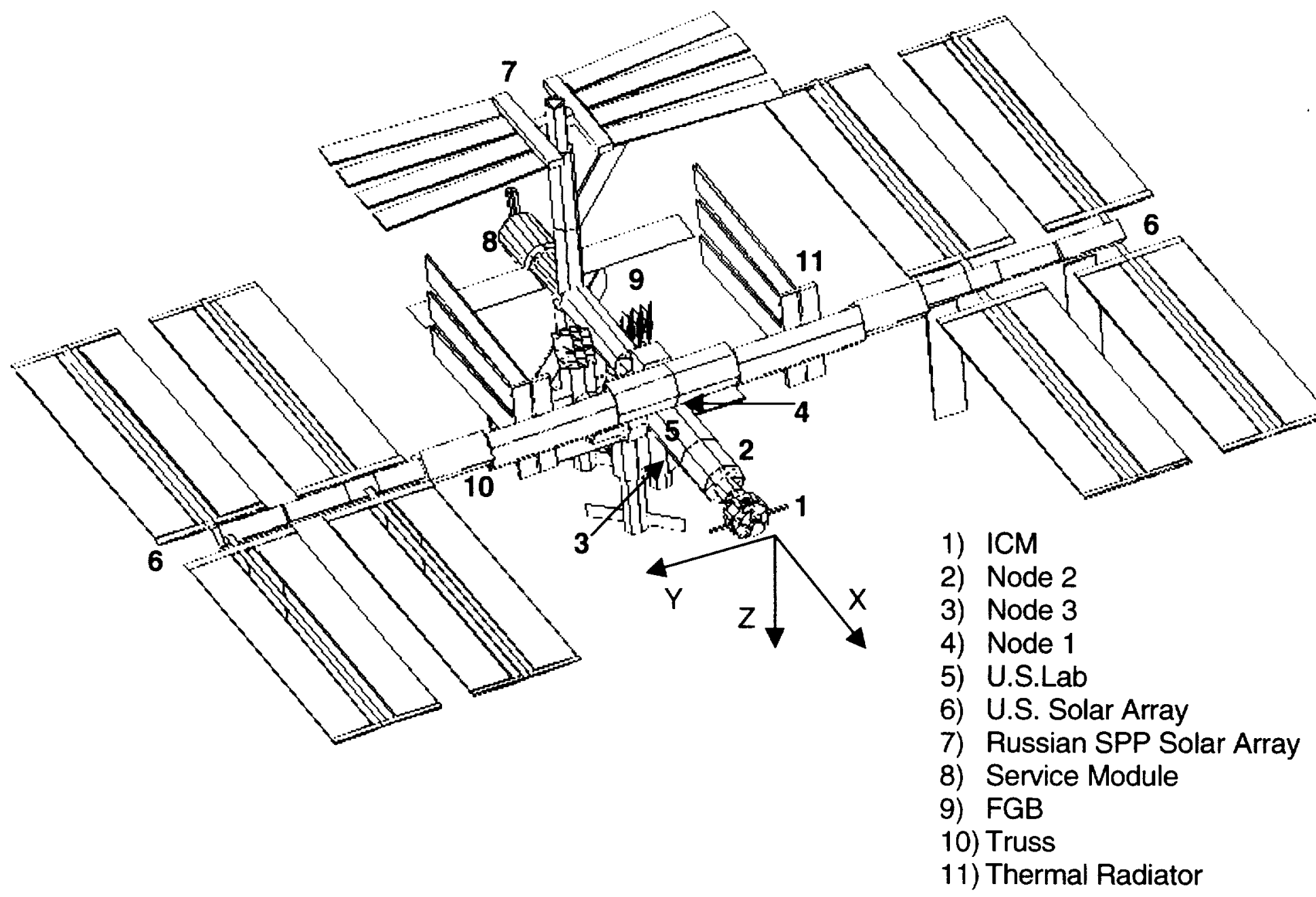

Figure 1: ISS Configuration, Element Locations and Coordinate System 


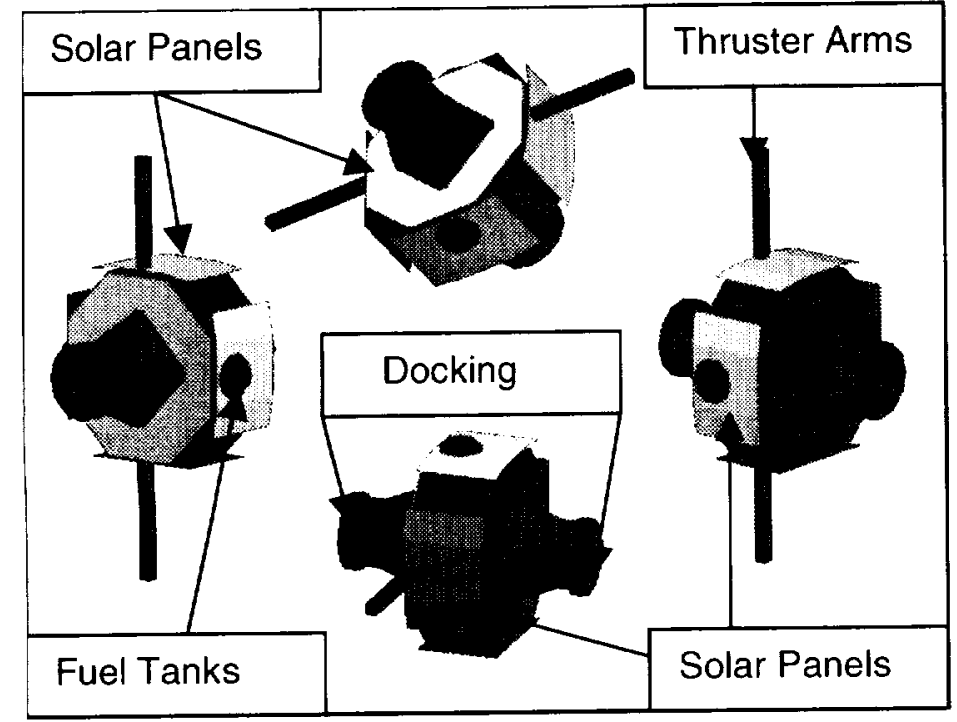

Figure 2: ICM Diagram

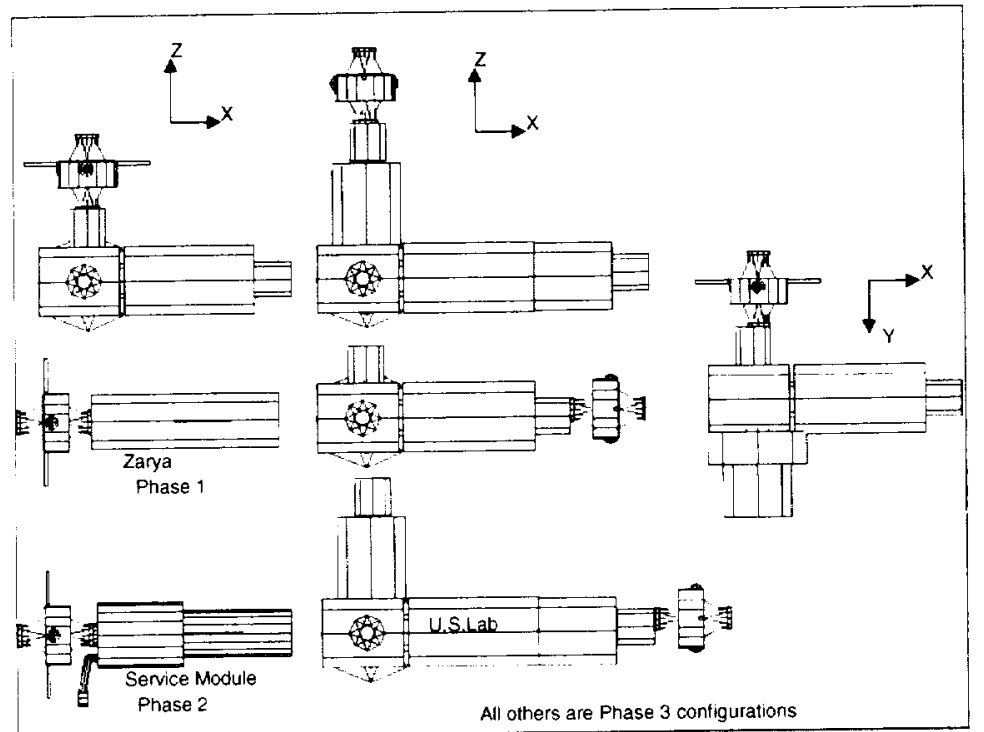

Figure 3: ICM Locations for Various Phases

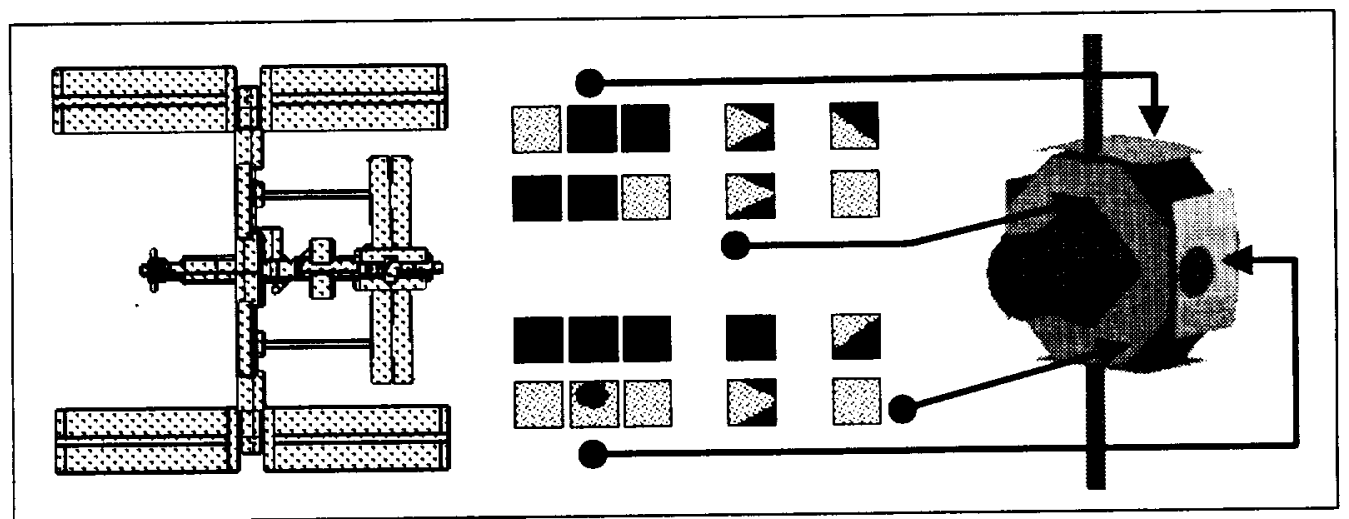

Figure 4 Typical SPACE Generated ISS Orientation and Shadowing Data

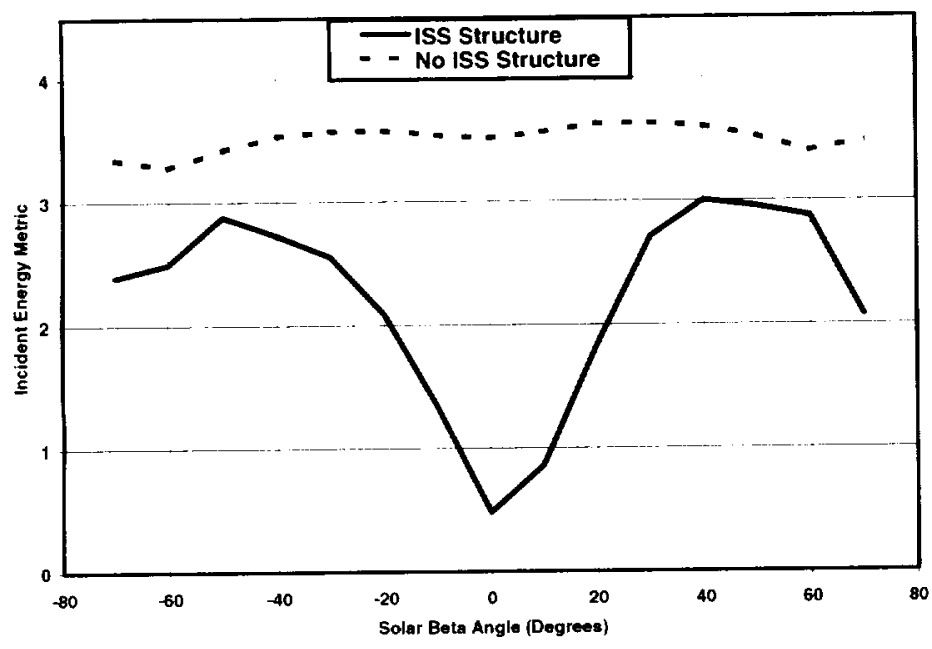

Figure 5: Incident Energy With/Without Shadowing

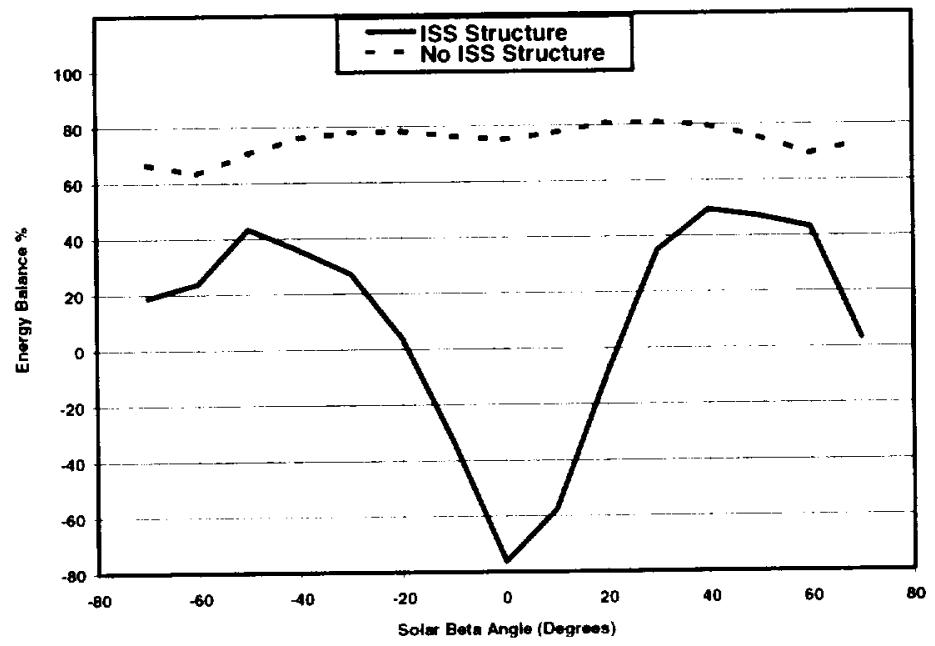

Figure 6: Energy Balance With/Without Shadowing 


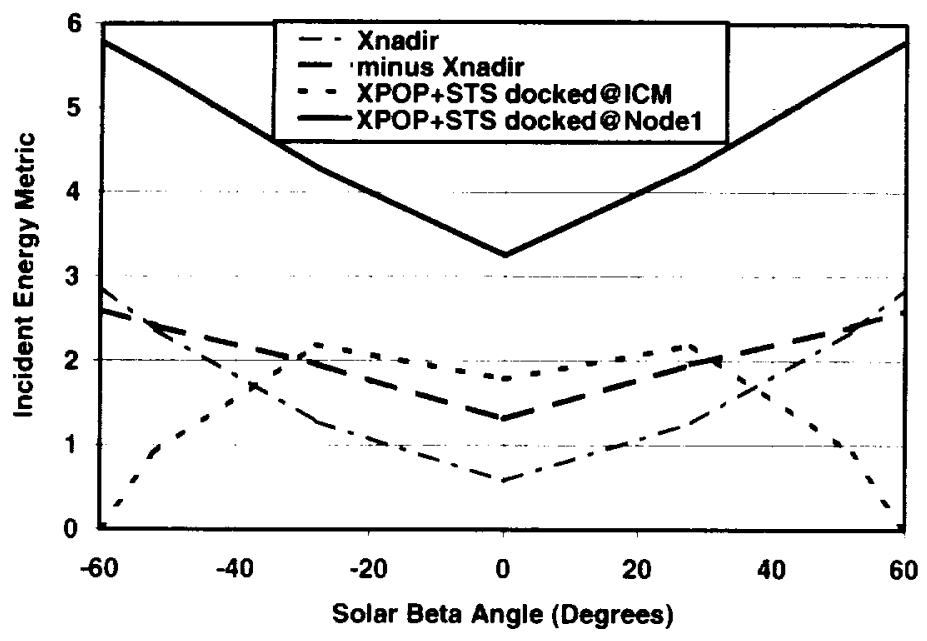

Figure 7: Phase 1/Incident Energy/Proximity Operations

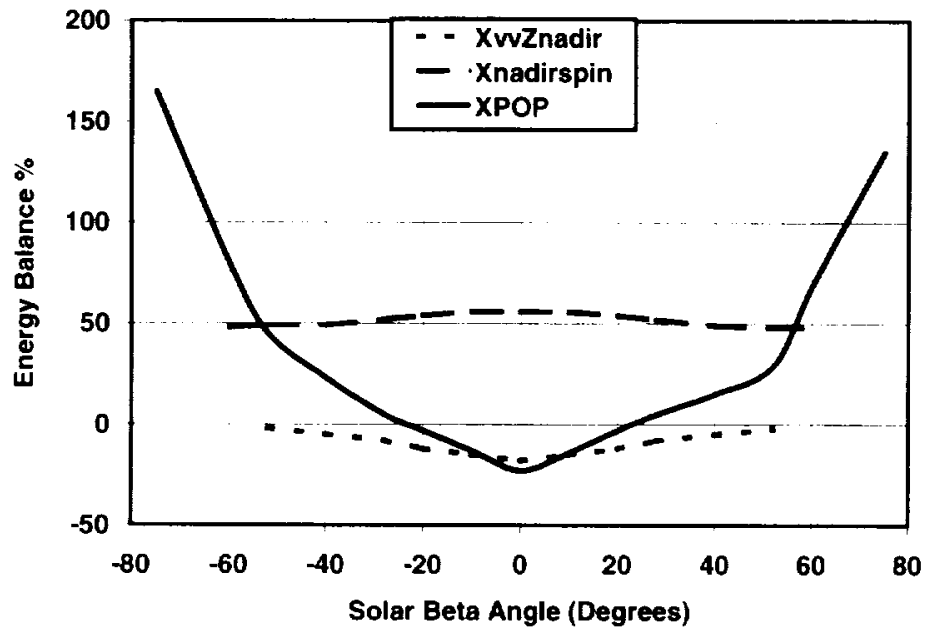

Figure 8: Phase 1/Energy Balance/Proximity Operations

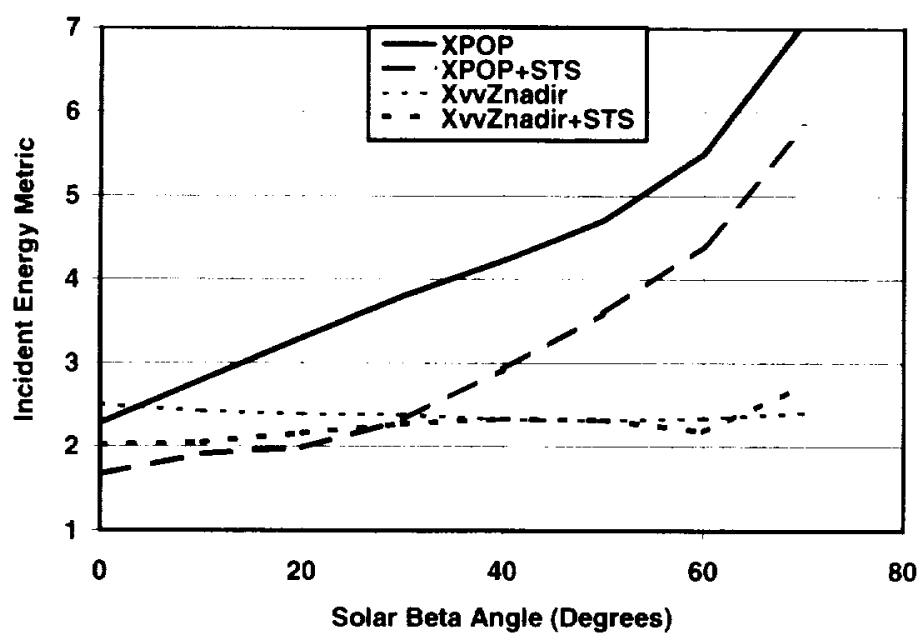

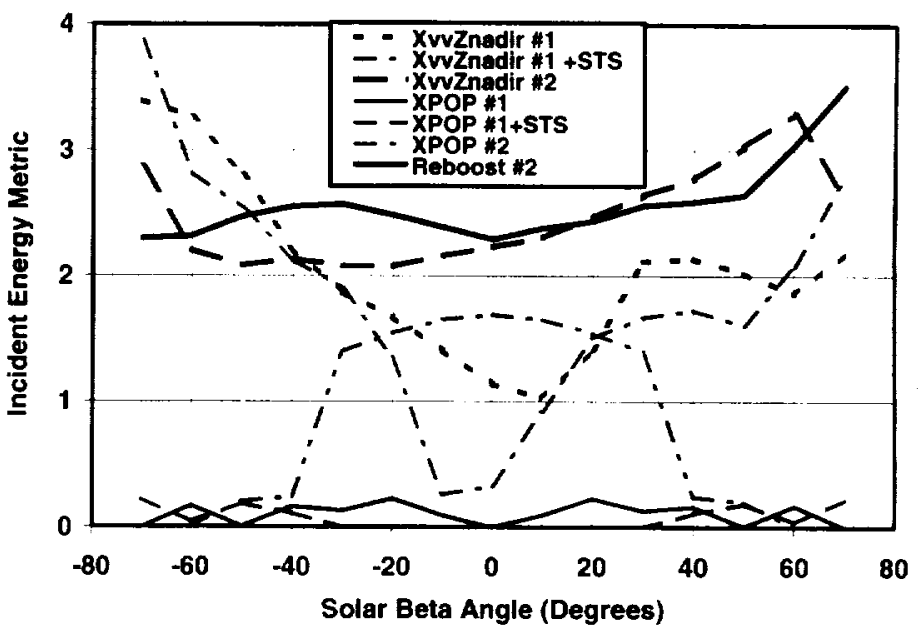

Figure 10: Phase 3/Incident Energy

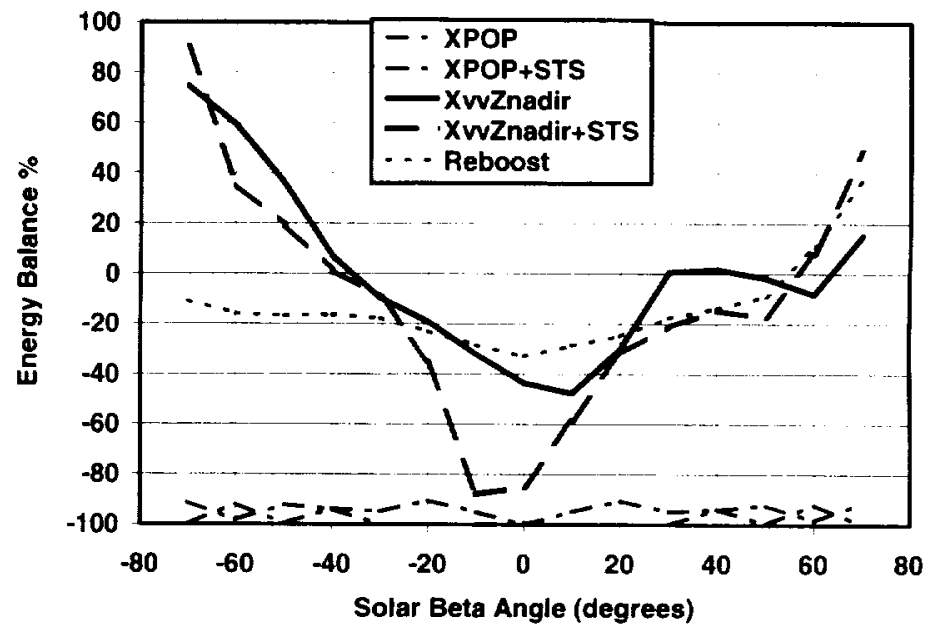

Figure 11: Phase 3/Energy Balance

Figure 9: Phase 2/Incident Energy 


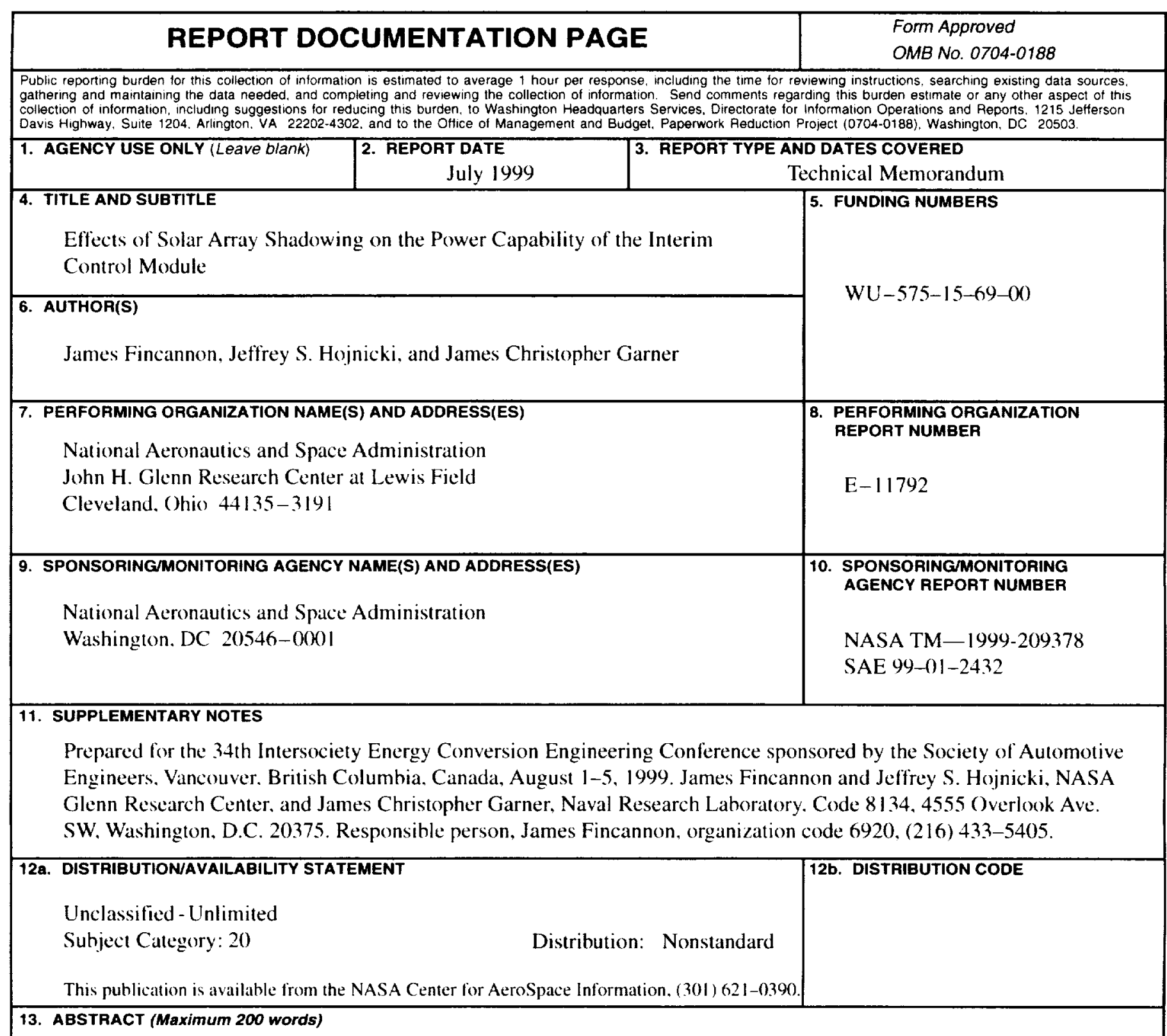

The Interim Control Module (ICM) is being built by the US Naval Research Laboratory (NRL) for NASA as a propulsion module for the International Space Station (ISS). Originally developed as a spinning spacecraft used to move payloads to their final orbit. for ISS. the ICM will be in a fixed orientation and location for long periods resulting in substantial solar panel shadowing. This paper describes the methods used to determine the incident energy on the ICM solar panels and the power capability of the electric power system (EPS). Applying this methodology has resulted in analyses and assessments used to identify ICM early design changes/options, placement and orientations that enable successful operation of the EPS under a wide variety of anticipated conditions.

\begin{tabular}{|c|c|c|c|}
\hline \multirow{2}{*}{\multicolumn{3}{|c|}{$\begin{array}{l}\text { 14. SUBJECT TERMS } \\
\text { International Space Station: Electric power; Shadowing: Models }\end{array}$}} & 15. NUMBER OF PAGES \\
\hline & & & $\frac{16}{16 . \text { PAICE CODE }}$ \\
\hline $\begin{array}{l}\text { 17. SECURITY CLASSIFICATION } \\
\text { OF REPORT }\end{array}$ & $\begin{array}{l}\text { 18. SECURITY CLASSIFICATION } \\
\text { OF THIS PAGE }\end{array}$ & $\begin{array}{l}\text { 19. SECURITY CLASSIFICATION } \\
\text { OF ABSTRACT }\end{array}$ & 20. LIMITATION OF ABSTAACT \\
\hline Unclassified & Unclassified & Unclassified & \\
\hline
\end{tabular}

